\title{
Across Fields: Sound, art and technology from an electromechanical perspective
}

\author{
JON PIGOTT \\ School of Art and Design, Cardiff Metropolitan University, 200 Western Avenue, Cardiff CF5 2YB \\ Email: jpigott@cardiffmet.ac.uk
}

\begin{abstract}
This article follows electromechanical technologies through different contexts of electronic and experimental music, sound art and kinetic art as well as through parts of their industrial development and application. The aim is to explore connections between these different fields which are often obscured by disciplinary and genre divides, and which are typically unrepresented by critical and historical accounts. The approach is influenced by the field of science and technology studies (STS, also science technology and society) where technical and cultural entanglements are seen as crafting particular truths, and where the method of following a technology across disciplinary boundaries is found. By taking this approach to identify connections between the areas of electronic music, sound art and kinetic art, new and rediscovered critical appraisals of the use of electromechanical technologies as tools in creative sound production are identified. These positions are then applied to a selection of contemporary practitioners who continue to work with and forefront electromechanical technologies within the fields of electronic music and sound art.
\end{abstract}

\section{FINDING HIDDEN LEADS}

Following hidden or outmoded technologies can uncover lost cultural connections and modes of creative practice. Reciprocally, following obscure or outmoded creative practices can help to better understand particular technologies. The challenge is staying on the trail across boundaries of discipline and genre description such as culture, technology, sound and art. The world of science and technology studies (STS) is helpful in showing how it may be possible and why it may be useful to follow technologies wherever they may lead in disciplinary terms. The field offers approaches to thinking about entanglements between culture, technology and history.

Sound art and kinetic art are two areas of creative practice that have struggled to achieve full critical and historical representation (see Cox 2011; Chau 2014). They both make important contributions to the world of electronic music and they are also two areas which have something to offer each other in terms of critical appraisal. How can these two apparently different and underrepresented areas be reappraised in the light of each other? This article takes the basic electromechanical assembly as a lens through which to explore particular examples of electronic and experimental music and sound art from the 1960s and 1970s, alongside concurrent examples of kinetic art which also feature sound and electronics. By doing this, new and rediscovered connections between the two fields emerge which can be used to critically inform a historical and contemporary context of electromechanical sound and sound art. This process also unveils something of the electromechanical assembly itself, its material characteristics and its role in creative practice and industrial and experimental technologies through the twentieth century. Establishing an 'electromechanical perspective' in this way is a useful starting point and one that is also informed by the STS methods.

Various accounts are helpful in developing an electromechanical perspective of sound art and electronic music, though it is often presented within a broader technological and creative context. For example, David Toop reviews some 400 years of sound art, automata and musical sculpture in 'Humans, Are They Really Necessary?' (Toop 1999) including many electromechanical examples, and Hugh Davies's entries on both sound art and electronic instruments in the New Grove Dictionary of Music and Musicians (Davies 2016a, 2016b) discuss electromechanical examples alongside wind-powered, hand-operated and purely electronic approaches. Elsewhere, historic perspectives from Jonathan Sterne (2003), Thom Holmes (2002) and Karen Bijsterveld (2008) also help to build a sense of an industrial electromechanical past, which includes projects such as the telegraph, the player piano and the Telharmonium.

For the purposes of this article, maintaining an electromechanical perspective involves only following technologies that combine and transduce between mechanical and electrical energy. In relation to creative practice, the focus is on creative methods that centre around or intervene in those technologies, and the energetic transduction which defines them. With this definition in mind, Alvin Lucier's Music on a Long Thin Wire (1977), David Tudor's Rainforest (1968 onwards) and Steve Reich's Pendulum Music (1968) will be explored for their electromechanical approaches and interventions, alongside works of sounding kinetic art 
from Jean Tinguely, Takis and Len Lye. Within this field the loudspeaker emerges as one particular electromechanical device with rich potential for creative intervention and exploration. Despite being an old, long-established technology, the loudspeaker remains an indispensable link between an electrified sound world and hearing, and creative methods that explore this indispensable link extend to contemporary examples of sound art. In this regard it is as if the electromechanical is both outmoded and future-proofed.

\section{ELECTROMECHANICAL ASSEMBLY AND ALIGNMENT}

Studying a technology closely can cause it to unravel. Internal technical assemblies, wiring and arrangements of components can begin to appear to hold together only through tenuous and overly complex systems when under close inspection. External social and commercial applications and uses of technologies help to keep technological black boxes neatly closed through control and careful management. Philosopher and STS scholar Bruno Latour shows this situation effectively through case studies of technologies including the diesel engine (Latour 1987: 104) and the Bell Company telephone network (Latour 1987: 125). Latour shows that the effective operation and widespread take up of a technology does not spring from a single moment of invention or discovery, rather it is a constant struggle between the relative successes, failures and alliances of various 'interest groups'. Interestingly, for Latour, these alliances and interest groups 'cut across the boundaries between human beings and things' (Latour 1987: 125). He describes how linking the east and west coasts of the United States with a single telephone line required a shift from mechanical to electrical signal boosters. The boosters were a 'missing link' (Latour 1987: 125) in an alliance between the Bell telephone company and the rest of the populous of the United States. But developing an operative electrical booster would require aligning the activity of physicists and physics laboratories with the needs of industry, and aligning the behaviours of electrons inside valves with the financial concerns of boards of directors. Through accounts such as these, Latour effectively shows the varied and complex relationships that exist between the human and the non-human within technological arrangements.

The moving coil loudspeaker is an example of a technology deeply embedded into our daily lives that can also appear to unravel through close inspection. Internally, the assembly at the heart of the loudspeaker falls apart through its very nature of being electromechanical. As the name suggests, this is a hybrid arrangement of electrical, mechanical and magnetic parts. Each of these elements has its own set of material concerns and exhibits a particular set of behaviours in relation to the others. By carefully assembling these materials, early loudspeaker designers of the late 1800s such as Earnst Siemens and Oliver Lodge (Chanan 1995: 39; Schoenherr 2001) would have encountered issues of loudspeaker design that continue to haunt the technology today. Coil resonance (the preference that a coil of wire has to respond to some electrical frequencies more than others), back EMF (a coil's tendency to generate electricity as well as respond to it when in a magnetic field), flexibility, material distortion and damping of the speaker cone (more resonances and material behaviours) and effective distribution of magnetic field are just some of the issues that need mitigating before a neat black box recognisable as a loudspeaker can be sent to market and put to use.

A much bigger problem facing the early proponents of the loudspeaker was that it was not so useful without the support of effective audio power amplification, a missing link that would not be available for some years after the moving coil assembly first emerged. Here, the future of the loudspeaker was dependent on the future of valve amplification which linked it to not only to the world of signal repeaters for telephone networks, but also to radio broadcasting, where amplification was already an embedded technology (Chanan 1995: 38). The world of radio broadcast, meanwhile, was keen to benefit from a more convenient and attractive listening experience than that of the in-ear headphones typical of early crystal sets. The sociotechnical concerns of moving coil loudspeakers, valves, amplification, radio companies and telephone networks were aligned. But with increased amplification, the large coil-driven speaking horns, which had begun to be marketed as pieces of furniture for the home, exhibited high levels of distortion. Here was a new missing link between the moving coil loudspeaker and every home that had a radio set. This missing link turned out to be a cone of stiff paper connected between the moving coil assembly and a baffle, vibrating sympathetically with the drive unit and producing loud and clear sound waves (a design move attributed to Kellog and Rice at General Electric, see Chanan 1995: 39). At this point careful alliances between amplification, marketing departments, radio networks, electrons and cardboard (among many other things) began to allow for the industrially produced, commercial, black boxed loudspeaker to become, quite literally, a dominant voice in the sociotechnological landscape.

\section{THE ELECTROMECHANICAL ASSEMBLY AS A CREATIVE TOOL}

By the mid-twentieth century, electromechanical technologies including the loudspeaker had become firmly embedded black boxes of sound and music production. This presents an interesting case study of how such technologies that enjoy an 'embedded' 
position can become less visible, particularly in the context of historical perspectives. Key developments in creative practice within the field of electronic music during the 1950s and 1960s are often located around the possibilities of sound itself being treated as a plastic material. The reversing, splicing, speeding up, slowing down, layering and spatial redistribution of sound was key in both popular and experimental music of the time. When considering these techniques, it is not necessary to acknowledge the synchronous and efficient motors, the artefacts and the resonances of electromagnetic transduction, the moving coils and the material distortions. The creative potential of magnetic tape recording allowed for the separation of the musical score from the musical outcome and, as John Cage put it, for an appreciation of 'the equivalence between space and time' (Holmes 2002: 78). When considering such creative possibilities as these, it is to be assumed that the fidelity of the electromechanical recording and reproduction systems is both good and reliable and that resonances, imperfections and distortions have been smoothed out for the benefit of human hearing and creative practice.

Thinking through the creative and cultural impact of the tape recorder and loudspeaker in this way is to place the electromechanical world as largely subservient to a world of high-quality sound manipulation and reproduction. This situation is well illustrated by Pierre Schaeffer's concerns with acousmatic and reduced listening for works created in what Ethan Rose describes as the 'idealised neutrality' of the studio (Rose 2013: 66). In this sense it is possible to replace the material and mechanical object of electromechanical technology with the more human-centric sonic object of listening and composition. In preference to the efficient manipulation and control of sound, the messy business of wires and magnets are carefully and effectively brought into line before being pushed out to the technological 'hinterland', the term that STS scholar John Law (Law 2004) uses to develop the idea of the embedded technological black box. ${ }^{1}$ These 'hinterlands' and 'black boxes' are accepted, refined, efficient, industrially produced, widely used but largely overlooked and ignored technological objects.

In contrast to this there are particular examples of electronic and experimental music and sound art from the 1960s and 1970s which do forefront the hidden electromechanical substrate of the technological landscape in the mid- to late twentieth century. Three examples to consider are Alvin Lucier's Music on a Long Thin Wire (1977), David Tudor's Rainforest (1968 onwards) and Steve Reich's Pendulum Music (1968). These examples

${ }^{1}$ This is not to say that all composers working within the acousmatic tradition specifically seek to hide electromechanical apparatus. For example, Francois Bayle's Acousmonium (1974) is a diffusion system of 80 loudspeakers of different shapes and sizes selected for their non-linear sonic characteristics.

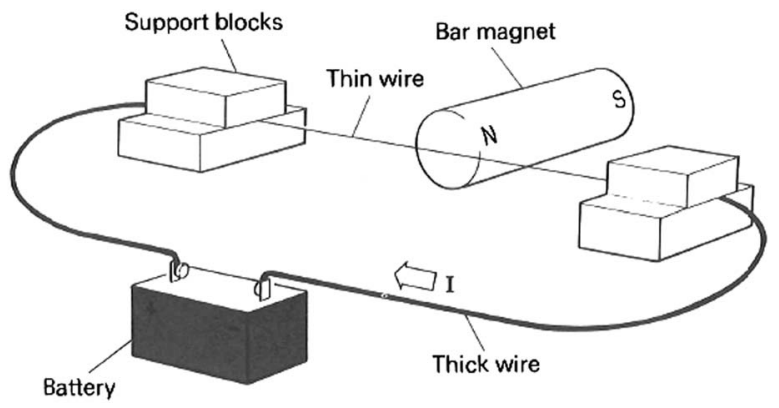

Figure 1. Electromechanical textbook illustration.

can, in part, be seen as belonging to a creative tradition of 'live electronics'. This is a mode of practice which enjoys a lineage to which Cage contributed and, as Nicolas Collins points out, embodies an ethos of 'music implicit in technology', an approach which served as a paradigm for much American electronic music of the 1970s (Collins 2007: 46). The pieces can also be seen as canonical examples of experimental music and sound art, though these genre descriptions are potentially problematic, partly suffering from a lack of full and effective critical representation (Cox 2011). The aim here is to review the pieces specifically in terms of their electromechanical credentials, highlighting their connections with the world of kinetic art, another area of creative practice which has been identified as problematic and underrepresented in historical and critical accounts.

Lucier's Music on a Long Thin Wire presents a simple assembly of materials demonstrating the basic electromechanical principle, appropriated for creative soundmaking. The piece is almost fully described by the textbook diagram in Figure 1, which shows a simple experimental electromechanical set-up. In Lucier's piece, however, the wire is mechanically excited by an alternating current that travels back and forth, supplied by a signal generator, rather than just a battery. This causes complex vibration and resonance, which is then amplified using contact microphones placed at either end. The piece exists as both a performance, with the frequency and level of the signal generator being adjusted by the performer, and a stand-alone installation where the material assembly of magnets, wire and electricity are left to perform on their own and in response to environmental factors such as temperature (Lely and Saunders 2012: 262). Lucier has described the piece as a 'deconstructed loudspeaker' (Lucier 1995: 186).

David Tudors Rainforest, similarly forefronts the loudspeaker and its inherent electromechanical sonic potential in a context that Collins describes as existing 'in the twilight zone between a concert and an installation' (Collins 2007: 46). The piece centres around customised loudspeakers described by Tudor as 'instrumental' or 'sculptural' (Driscoll and Rogalsky 2004: 28). These devices appropriate the electromechanical transduction element of a loudspeaker to resonate objects such as 
cartwheels, bedsprings and oil drums, among various other objects, in installations and performances which were realised by Tudor's group Composers Inside Electronics throughout the 1970s. In Rainforest audience members are able to move through the space occupied by the sculptural speakers listening to the resonant characteristics of the objects which may be directly audible acoustically, or re-amplified through other loudspeakers. Tudor has described the piece as developing from the notion that the loudspeaker should have a voice which is not just an instrument of reproduction but is an 'instrument unto itself' (Tudor 1988).

Pendulum Music, the third example which explores the electromechanical, is often discussed in terms of its minimalist credentials and as an early example of Steve Reich's long-established interest in relational time phased processes. In the piece, performers release microphones, suspended by their cables, such that they swing in a pendular motion above loudspeakers causing short bursts of audio feedback. These short bursts develop a complex rhythmic relationship to each other, then gradually increase in duration as the microphone pendulums slow down, ultimately arriving at a continuous howling as the pendulums come to rest. Interestingly, writing in 1969, Reich claimed that 'whether a musical process is realised through live musical performance or electromechanical means is not finally the main issue' (Reich 1969: 305), also stating that when working with electromechanical sound equipment it is natural to think about such musical processes as are used in Pendulum Music (Reich 1969: 305).

These three examples clearly fit neatly into Collins's notion of a compositional approach that sees music implicit in technology. The lack of emphasis on human performers reflects this, with Music on a Long Thin Wire and Rainforest both appearing as stand-alone installations and Pendulum Music using performers only for the initial energetic impulse that begins the piece and to switch off the amplifiers at the end. The technology is centre stage. This is something that Ethan Rose understands through the examples from Lucier and Reich as a translation of the 'tools of the recording studio into a visible bodily presence', also describing the pieces as 'object based sound installations' (Rose 2013: 66).

The foregrounded technologies in these examples are electromechanical, and the works exhibit an aesthetic concern with electromechanical behaviours. Lucier's piece deconstructs a loudspeaker not only in practical and experimental terms but also by amplifying the sonic irregularities of the electrical, mechanical and magnetic assembly. Similar concerns are identifiable in Tudor's comment that the loudspeaker should be allowed to be an 'instrument unto itself', something active and present, rather than a passive transmitter of an electrified sound world under human control.
Tudors sculptural speakers emerged from his experimenting with a hobby project from a 1966 edition of Popular Mechanics magazine which described how to 'build a fantastic coneless loudspeaker' (Driscoll and Rogalsky 2004: 26). It is interesting to consider how such hobbyist and DIY approaches can undo established commercial technologies, in this case reintroducing material distortions to the electromechanical signal path. Pendulum Music, though less obviously concerned with the actual disassembly and representation of the internal electromechanical process, is actually the purest reflection of electromechanical sound-making. The piece is a no input, feedback-based system whose audible output is defined entirely by the resonant behaviours of the speaker and microphone relationship, modulated by the swinging of the pendulum-microphones. All three examples in this context are united through a concern with the objects, materials and technologies of electromechanical sound-making, and through themes of energetic transduction, resonant behaviour, and movement.

It is from this perspective and through these themes that strong connections with some examples of kinetic art begin to emerge. Following these connections and exploring some of the critique surrounding kinetic art leads to distinctions which reflect usefully on creative electromechanical approaches in sound. This is worthwhile particularly as approaches that forefront the electromechanical continue to emerge from practitioners today as well as and alongside neat black boxed electromechanical technologies such as the loudspeaker, which remain widely used and deeply embedded in commercial techno-culture. Three examples of kinetic art from the artists Takis, Jean Tinguely and Len Lye, all from 1960s, set the scene for these connections, and a broader view of the context of kinetic art at this time will help to draw out distinctions between approaches identified as robotic and process driven within the field.

The Greek artist generally known as Takis (first name Vassilakis), who spent much of the 1950s and 1960s working between Europe and the United States is a sculptor, often regarded as kinetic, interested in the 'unseeable energies of nature' (Andersen 1968: 23) and in particular magnetism and electromagnetism. Since the 1950s Takis has also included sound in his work. These interests in magnetism, electricity and sound were brought together in 1963, in a collaboration with composer Earle Brown titled Sound of the Void where electromagnetic fields were used to energise a needle to strike a string, creating a repetitive musical refrain. Around this same time a series of similar electromagnetic and kinetic sound works emerged under titles including Magnetic Pendulum Musical (1965), Telemagnetic Musical (1966) and Electro-Musical Relief (1966) all of which appropriated the kinetic sound-making possibilities of electromagnets, fixed 
magnets and resonant wires under tension, sometimes strung over a taught canvass as a kind of soundboard. These works were a development of similar magnetic themes explored in earlier works such as Magnetic Ballet (1960) and Telemagnetic Sculpture (1959). Through all these works Takis demonstrates a concern for an almost objective presentation of materials and their energetic behaviours (sounding or otherwise), stating 'I follow the indications of the materials, I do not dominate them' (Takis quoted in Burnham 1968: 271).

Len Lye, whose work from this time is also broadly recognised as kinetic art, similarly worked with magnetism, mechanical movement and sound in The Loop (1963, also titled Universe). This is a 22 foot strip of polished steel formed into a band, which is both tethered and energised by a strong electromagnet inside a plinth. The steel emits tones and harmonics as it rocks and wobbles around on its plinth, occasionally lurching up high enough to strike a ball suspended above it causing a different set of sounding behaviours within the steel band. The piece, described by critic Jack Burnham (1968: 270), 'dances to a weird quavering composition of its own making' and serves as a good example of how Lye's work is controlled by the 'dynamical properties of the materials used' (Burnham 1968: 274).

Swiss sculptor, Jean Tinguely, often regarded as the father of kineticism, has a large body of work that could broadly be regarded as electromechanical through its use of motorised movement. Sound has also been a concern of Tinguely's since at least the 1950s in pieces such as his Meta Mechanical Sound Reliefs (1955), which involved the production of sound through the kinetic striking of saucepans, jars, glass funnels and wine glasses by light hammers (Hulten 1987: 28). Later development of these pieces came with the much larger Meta-Harmonie series (1979-85). These industrial-sized assemblages of steel cogs and wheels, wires, belts and musical instruments emit a cacophony of mechanical noise alongside occasional pitched or percussive sounds. Of particular interest from the electromechanical perspective, however, are Tinguely's Radio Sculptures (1962; Figure 2). These are deconstructed but operative radios emitting live broadcast sound which was kinetically modified by electromechanical devices fitted to their tuning dials and volume controls. The combination of the electromagnetic induction of radio waves, the exposed speaker and amplifier circuitry and the motorised, kinetic adjustment of the dials represents an exploration of electromechanical transduction and behaviour.

These three kinetic artists not only use electromechanical technologies in their work but also, like the three examples from Lucier, Tudor and Reich, choose to foreground and draw out aesthetic concerns that can be tied to the basic electromechanical assembly.

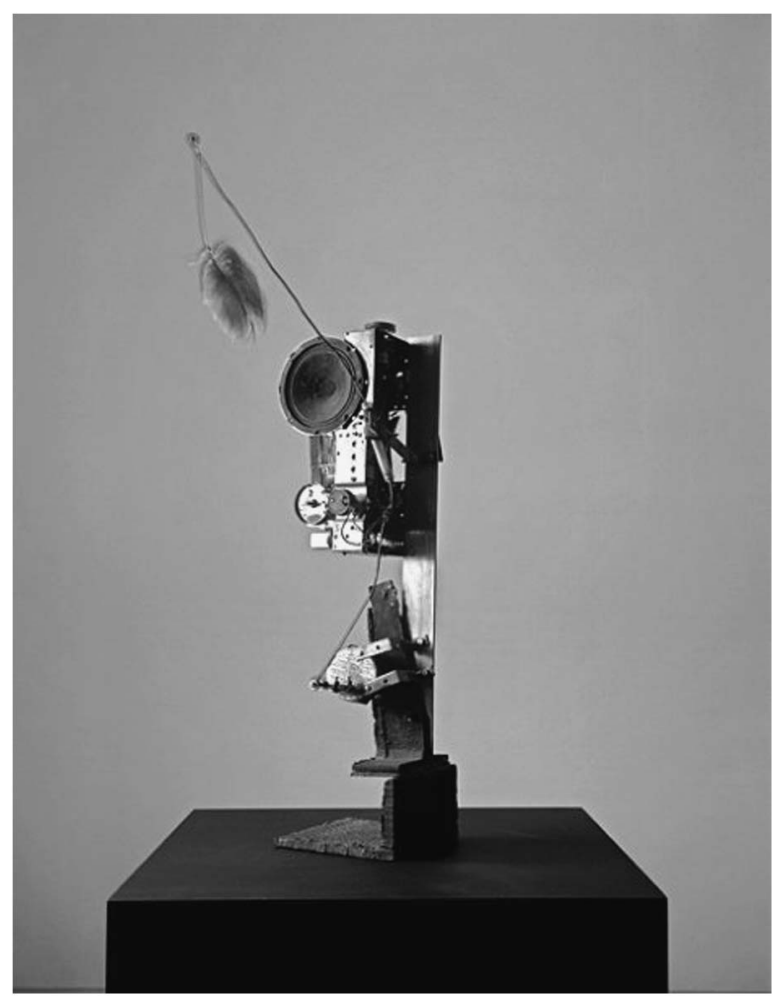

Figure 2. Jean Tinguely, Radio Sculptures (1962).

Through a primary concern with magnetism, Takis is working with component parts of the electromechanical assembly and through his various arrangements and rearrangements, movement and sound emerge. Takis's interest in the 'unseeable energies of nature' can be aligned to Pontus Hulten's description of Tinguely's "mastery of "immaterial" materials' (Hulten 1987: 121), and Jack Burnham's description of Lye's work appearing as 'half material and half pure energy' (Burnham 1968: 269). All these descriptions could equally relate neatly to a technical assembly whose very nature is based upon energetic transduction between hard mechanical materials and invisible electrical and magnetic materials.

The emphasis within these kinetic pieces on something that is somehow half material and half unseeable, energetic or immaterial, is also reflective of Lucy Lippard's description of the the art world of the 1960s as undergoing a 'dematerialisation' (Lippard and Chandler 1967). At this time the established modernist mode of sculpture as a fixed, material specific, static object on a plinth was beginning to give way to more expanded and conceptual approaches which could include the use of light, sound and performance for example. Technological systems played an important part in these developments. The electromechanical condition reflects this dematerialisation within the art world in metaphorical and practical terms by belonging to a world of both hard materials and soft energetic concerns. The examples from Takis, Tinguely and Lye 
from the 1960s illustrate these developments by foregrounding the electromagnetic assembly.

\section{THE ELECTROMECHANICAL ASSEMBLY AS A REPRESENTATIONAL TOOL}

Kinetic art has struggled to fully establish a position for itself in more recent art history and theory. Despite the novel approaches described through the work of Takis, Tinguely and Lye, which point towards a future of dematerialised and expanded art practice, kinetic works remain, to a greater extent, connected to an older, modernist machine aesthetic with strong ties to Futurism and Constructivism. Christina Chau describes how Jack Burnham, an influential art critic of the 1960s who was also once a kinetic sculptor himself, developed a dissatisfaction and antipathy for kinetic and electromechanical sculpture in his writing. Burnham deemphasised the relevance of kinetic works in favour of developing a general theory of systems art which had the capacity to encompass a broader range of approaches, including conceptual, socially and environmentally engaged or data-driven artworks. Chau considers this move by Burnham an attempt to 'sequester the theory and practice of movement in art away from postmodern aesthetics' (Chau 2014: 64). Writing in the late 1960s, Burnham certainly struggled to align kinetic art, which often appeared as plinth mounted and object based, with more emergent trends in art practice. Paradoxically though, one of the best documents of kinetic art from the time is Burnham's Beyond Modern Sculpture (1968). Now out of print, the book is divided into two sections titled Sculpture as Object and Sculpture as System.

Sound art and sound sculpture are modes of practice which have also struggled to be properly and fully represented through a historical and critical perspective. Cristoph Cox describes how, following the 1960s, a philosophical programme that favoured the analysis of images and texts bolstered practices such as conceptualism, dismissing notions such as non-discursive perception and materiality (Cox 2013). Cox further claims that as a result:

sound art was left without a robust theoretical basis or mode of apprehension and was thus relegated to a minor status, at best an adjunct to music, at worst a naïve or retrograde incursion into the visual arts. (Cox 2013)

Vadim Keylin highlights a similar problem of representation for the more specific genre description of sound sculpture. Considering the work of Harry Parch alongside examples including Tinguely, Peter Vogel and the instruments of the Baschet brothers, Keylin claims sound sculpture is excluded from sound art discourse for being 'so unmodernly modernist', and thus remains 'problematic and underexplored' (Keylin 2015: 182). Again the associations with objects, materials and plinths seem to keep the field tied firmly to modernist values. Keylin seeks to re-establish links between music and sound sculpture in order to help an understanding and analysis of the field.

These underrepresented and 'problematic' areas of kinetic art and sound art or sound sculpture relate and reflect usefully on each other. The challenge is bringing them together meaningfully across divides of genre descriptions which are already problematic. Terms such as 'sculpture', 'music' (experimental, electronic or otherwise), 'art' and 'sound' can seem both too exclusive and at the same time too general to help with this. An electromechanical perspective weaves a narrative through and across different fields, drawing together specific and interdisciplinary concerns for certain works across discourses and genre descriptions. The approach of following a technology through and across disciplines, genres and contexts is reflective of the approach taken by science and technology scholars such as Latour. STS represents a tradition where artefacts are followed across boundary descriptors such as society, economics, culture, science or technology (Latour 1987; Law 2009).

\section{PROCESS}

One such interdisciplinary theme which draws together all the examples presented here is that of 'process'. In this context, process is to be understood as a creative method that relinquishes some element of control by the human artist/composer and allows other human or non-human elements of the creative process to play a part in the natural unfolding of events and co-creation of the work. With connections to Umberto Eco's idea of the 'open work' (Eco 1959) and Cage's experimental and indeterminate compositional approaches, the terms 'process art' and 'process music' are loosely recognisable genre descriptions of work that began to appear during the 1960s from a number of practitioners including those discussed here. A process sensibility is reflected in Takis's claim that he tries not to dominate materials and in Burnham's description of Lye's work as being controlled by the dynamical properties of the materials used. Burnham further describes the process-led creative approach as creating a 'situation in which things can happen rather than an object per se' (Burnham 1968: 271). As Nyman points out, this is also an appropriate way of thinking through many pieces from Lucier's oeuvre, and Music on a Long Thin Wire clearly fits this description (Nyman 1999). Tudor's Rainforest, whose score is simply a generalised circuit diagram showing an arrangement of components and a position for 'objects to be transduced', can also be viewed as process-led. Steve Reich is possibly one of the names most readily associated with the idea of process music through his early works from the 1960s, which he discussed in his 1969 essay 'Music as a Gradual Process' (Reich 1969). 
Reich makes an important distinction between processes that run in the moment of the music, such as with Pendulum Music, and processes which are not discernable by an audience in their moment of reception as, for example, with Cage's use of the I Ching to determine a score for later performance in Music of Changes (1951). This concern for a 'compositional process and a sounding music that are one and the same thing' (Reich 1969: 305) is useful in forming connections between kinetic sculpture and some experimental music and sound art of an electromechanical nature. The examples from Lucier, Tudor, Reich, Takis, Tinguely and Lye considered here are all real-time processes which run in the moment of the sounding outcome. The point at which the artist/composer creators have let go of the work, and the nature of the unfolding events that make up the work in terms of its sounding and kinetic activity, are tangible in the moment of reception. The music and the method by which the music is coming into being evolve simultaneously in a way that is representative of some of the non-human materials at play. The clear physical presence of the material behaviours which make up the creative process also help to define these examples in relation to other more hidden, computational approaches to generative systems within sound and music (e.g. see Gogins 1991).

The musical examples from Lucier, Tudor and Reich make either minimal, optional or no use of human performers and this helps to clarify where the materially led process begins and where the music implicit in the technology can emerge. The examples of kinetic sculpture from Takis, Tinguely and Lye carry no historical baggage of human performance and as such present their non-human, real-time processes as a development of the static object of art. All the examples presented across the sculptural and musical field share a concern with a core, real-time electromechanical process running in the moment of the sounding outcome through a kind of non-human material performance. In relation to some mid-1960s kinetic sound works such as these, Toop usefully describes how 'as resonant or amplified solids move and interact, activated by unpredictable systems, the patterns of sound they create take on the drama of natural emergent phenomena' (Toop 1999: 125).

Electromechanical assemblies are by their very nature processes that exhibit the live animation of materials and the transduction of energies in real time. As shown, this is partly what causes problems for designers of loudspeakers and other such technologies when some of these live processes such as resonances, distortions and back EMFs are a little bit too live and need pulling into line, damping and quieting. From a creative position these sometimes unpredictable processes add to the sense that the artist/composer has relinquished control and the work is free to voice the unexpected behaviours of the electromechanical.
The antithesis to this process sensibility is described by Burnham through reference to automata and robotic technologies. This is a valuable differentiation for creative electromechanical approaches of all kinds and represents one area where Burnham concedes that some kinetic art of the 1960s offers something other than a modernist machine aesthetic. Burnham describes how the word 'robot', first coined by writer Karel Capek, comes from a Czech word meaning 'forced labour', and implies a lack of autonomy and free will (Burnham 1968: 202). He uses the automata and mechanical music boxes popular in the nineteenth century as a good example of this. Here, materials were formed and bent into machines that would replicate and reliably carry out a human programme of action such as music reproduction. Player pianos (mechanical or electromechanical) are further examples of once highly popular devices which similarly were made to accurately reproduce a human centric musical agenda in an age before widespread sound recording or electronic music sequencing. Despite some of these technologies seeming quaint, technologically and materially intriguing and unusual today, they belong in large part to an agenda of programmatics and control. Bijsterveld describes how these early mechanical and electromechanical music technologies served to 'defend the genius of a composer against the intrusions of a performer' (Bijsterveld 2008: 156) by etching the 'great minds of composers into the machine' (2008: 153) and it is possible to see how other electromechanical technologies such as the tape recorder and loudspeaker can be further extensions of this. These positions represent a technological and creative approach which seeks to control rather than celebrate the agency and behaviour of nonhuman materials. Following Burnham, these approaches can be thought of as 'robotic' in contrast to the previously outlined, process-driven approaches of the examples in section 3 .

This distinction from Burnham supported by Bijsterveld's insights form a useful tool in understanding the more recent trajectory in electromechanical approaches to sound and music. In reciprocation, Reich's clear distinction between compositional processes that run in the moment of reception and processes which are not may be a useful tool in discerning within a range of artworks that Burnham broadly aligned to his notion of 'systems art'. Ultimately, perhaps 'kinetic' was not and is not the most useful distinction when looking at creative work that deals with localised systems and material processes. A comprehension of the live energetic transfer within an artwork does not necessarily require brute movement. An aesthetic experience derived from the apprehension of localised materials, energy sources and their relationships can be gained through other means such as sound for example. 


\section{CONTEMPORARY ELECTROMECHANICAL APPROACHES IN SOUND}

Fortunately, a lack of critical acknowledgement or literature on kinetic and electromechanical sound art and experimental music has not left the field too bereft of practitioners choosing to work in this way. Since the 1960s there has been continuous creative activity within this area including from people who began making work concurrent with some of the examples discussed here. The work of kinetic sound artist Max Eastley, the large mechanical musical theatrics of Godfried-Willem Raes and the Logos Foundation, Pierre Bastien's kinetic installations and performances, Trimpin's sound sculptures, Martin Riches's music machines and sound installations, Gordon Monahan's works for loudspeakers, kinetic sculpture and piano and the continued work by Tudor's group Composers Inside Electronics (CIE) all represent a connection back to the original wave of interest in electromechanical approaches of the 1960s and early 1970s. Meanwhile another generation of practitioners has emerged from a context of easily available and ubiquitous digital technologies, who also choose to focus on the creative potential of the electromechanical. Peter Bosch and Simone Simons's large installation-based music machines and sound sculptures, Daniel Wilson's 'miraculous agitations' (Wilson 2012) of objects for sonic effect, Andrea Valle's Rumentarium Project, described as 'acoustic computer music' (Valle 2013), Ethan Rose's object-based sound installations, Shawn Decker's motorised sound art installations, Jim Murphy, Ajay Kapur and Dale Carnegie's musical robotics (Murphy, Kapur and Carnegie 2012), Zimoun's minimalist kinetic installations and Felix Thorn's machines represent a few such examples. It is possible to consider this broad range of contemporary creative practice in terms of the distinctions identified between processdriven and robotic creative approaches. This is not to suggest that hard lines of genre descriptions should be drawn up unnecessarily. Equally though it is not helpful to consider all work to be qualitatively the same just because it makes use of electromechanical or kinetic approaches.

Much of Bosch and Simons work seems to delight in the unexpected behaviours of materials when excited by electromechanical events. For example, their piece Krachtgever (1994) consists of a large structure of shipping crates, each filled with rattling resonant materials and conjoined by springs to the adjacent crate (Figure 3). This structure is vibrated and excited by motors running at different frequencies, driven by 'musical phrases' (Bosch and Simons 2005: 106). The physically complex, live and resonant system exhibits a process-driven sensibility where the exact sounding behaviour of the shipping crates is unknown in advance.

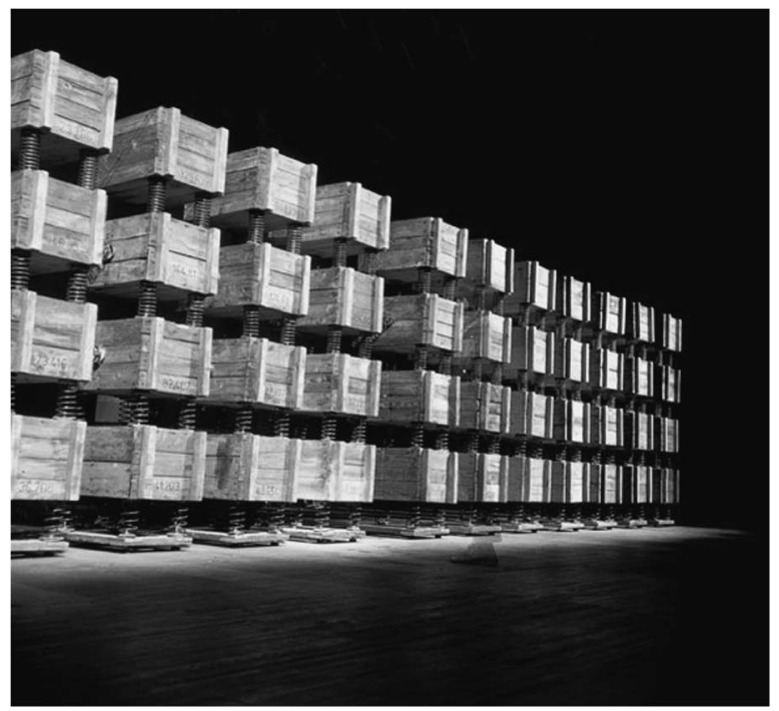

Figure 3. Peter Bosch and Simone Simons, Krachtgever (1994).

The same values are present in Andrea Valle's Rumentarium project which uses small DC motors to excite discarded objects and resonant boxes of materials (seeds, rice, plastic beads). Also Daniel Wilson's physical vibrating systems at the end of and as part of an electrical signal chain take a similar approach to allowing non-linear and unpredictable material behaviours into the creative process.

On the other hand, examples from Jim Murphy and Trimpin could be identified as exhibiting robotic tendencies. Trimpin's Conlon in Purple (1997) consists of a large installation of resonant wooden and metal bars excited by an electromechanical mallet system. The installed objects can be played through audience interaction or left to reproduce pre-recorded musical sequences. The sounding behaviour of the objects is reliable, repeatable and playable. Similarly, Jim Murphy's Bacchus (2012) described as a modular kinetic sound sculpture and new musical instrument uses a small motor, driven by a MIDI interface to excite a tuned wine glass. Like other examples of Murphy's work there is a strong theme of musical robotics here. Both Murphy and Trimpin are concerned with localised sounds which offer more than standard electronic or digital synthesis techniques can offer. There is also a concern with the spatial distribution of sound sources beyond that which standard industrially produced loudspeaker production can offer. But the pieces are not driven only by an electromechanical process sensibility, they are concerned with compositional, organisational and interactive possibilities which are pre-arranged or performed by humans. A similar judgement could be made of Felix Thorn's machines which combine the electromechanical technologies used in orchestrion machines with an electronica aesthetic. In these examples the sound is produced in a novel 
electromechanical way using robotic techniques of very precise and accurate control to enact a human scheme of sound organisation.

The kinetic machines that Pierre Bastien makes for performance and installation represent an interesting case for these distinctions between robotic and processdriven electromechanics. While some of Bastien's home-made electromechanical devices seem to resemble elements of automata and robotic music boxes, in action the musical precision and sounding outcome is less controlled and less reliable. Rather like a toy version of Tinguely's large cacophonous Meta-Harmonie series, Bastien's creations, often made from found objects and toy Meccano have their own musical agenda. Sounding events happen in repeating cycles and through ad-hoc creations that resemble early mechanical music sequencers, but without the relational accuracy required to establish reliable 'musical' loops. The sounding outcome is more a kind of unfolding process of mechanical time and material events. In performance Bastien's own live trumpet accompaniment meets this machine activity half way in an example of an ensemble of the human and non-human in creative collaboration.

\section{SUMMARY}

A large part of STS is concerned with issues of representation and power structure within human and technological entanglements. John Law's notion of a 'method assemblage' (Law 2004: 41) explores the idea of epistemologies and technologies combining in methodological black boxes which amplify particular truths and attenuate others; the human and non-human conspiring together to paint a particular picture of the world. With this sensibility it is possible to view a philosophical programme that favoured the analysis of images and texts after the 1960s, and a systems art that looked towards a burgeoning data-driven future as conspiring to obscure object-based, kinetic and electromechanical approaches in sound, music and art. Furthermore, exciting new possibilities of manipulating sound as a plastic material in either the digital or analogue domain requires the efficient control and black boxing of electromechanical processes which remain present but hidden, their inherent technological musicality made as linear and as predictable as possible.

Studying a technology closely and following it across disciplinary borders reveals something of the nature and application of its assemblage in ways that combine and challenge standard divisions between the technical and the cultural. By taking this approach with the electromechanical assembly, it has been possible to make and remake historical connections between sound art, kinetic art and electronic and experimental music, connections which also have contemporary relevance. One very useful result of this is a distinction between creative methods identified as process driven on the one hand and more robotic on the other. Creative electromechanical approaches in sound can celebrate a material agenda in processdriven works that are unpredictable and exhibit emergent behaviours within their technological systems, behaviours described by Toop as the 'drama of natural emergent phenomena' (Toop 1999: 125). Alternatively, approaches can celebrate the robotic control of materials through the intrigue and excitement of complex technological systems which respond effectively and accurately to programming and interaction. Such approaches can sometimes reference the technologies of orchestrion machines or player pianos. These important distinctions between processorientated and robotic approaches can easily be muddied as both often present sound outside of the context of standard industrial loudspeaker listening. Through the use of modified loudspeakers, motors and other electromechanical actuators both approaches benefit from the unmediated timbres and spatial displacement of 'live' electromechanical sound events.

The underrepresented and 'problematic' fields of kinetic art and sound art have something to offer each other in terms of a critical understanding of their histories and their possible futures. Uncovering these connections requires approaching the fields through paths other than the established cultural, historical and genre-defined ones. An STS-influenced electromechanical perspective is one possible approach to looking and listening back as well as forward.

\section{REFERENCES}

Andersen, W. 1968. Takis: Evidence of the Unseen. Cambridge, MA: MIT Press.

Bijsterveld, K. 2008. Mechanical Sound: Technology, Culture and Public Problems of Noise in the Twentieth Century. Cambridge, MA: MIT Press.

Bosch, P. and Simons, S. 2005. Our Music Machines. Organised Sound 10(2): 103-10.

Burnham, J. 1968. Beyond Modern Sculpture. London: Penguin Press.

Chanan, M. 1995. Repeated Takes: A Short History of Recording and It's Effects on Music. London and New York: Verso.

Chau, C. 2014. Kinetic Systems: Jack Burnham and Hans Haacke. Contemporaneity 3(1): 62-76.

Collins, N. 2007. Live Electronic Music. In N. Collins and J. d'Escrivan (eds.) The Cambridge Companion to Electronic Music. Cambridge: Cambridge University Press.

Cox, C. 2011. Beyond Representation and Signification: Toward a Sonic Materialism. Journal of Visual Culture 10(2): 145-61.

Cox, C. 2013. Sonic Philosophy. http://artpulsemagazine. com/sonic-philosophy (accessed January 2016).

Davies, H. 2016a. Sound Sculpture. Grove Music Online. Oxford University Press. www.oxfordmusiconline.com (accessed November 2016). 
Davies, H. 2016b. Electronic Instruments (I-IV). Grove Music Online. Oxford University Press. www.oxfordmusiconline. com (accessed November 2016).

Driscoll, J. and Rogalsky, M. 2004. David Tudor's Rainforest: An Evolving Exploration of Resonance. Leonardo Music Journal 14: 25-30.

Eco, U. 1959. The Poetics of the Open Work. In C. Cox and D. Warner (eds.) Audio Culture: Readings in Modern Music. London and New York: Continuum, 2004.

Gogins, M. 1991. Iterated Functions Systems Music. Computer Music Journal 15(1): 40-8.

Holmes, T. 2002. Electronic and Experimental Music. London: Routledge.

Hulten, P. 1987. A Magic Stronger Than Death. London: Thames and Hudson.

Keylin, V. 2015. Corporeality of Music and Sound Sculpture. Organised Sound 20(2): 182-90.

Latour, B. 1987. Science in Action. Cambridge, MA: Harvard University Press.

Law, J. 2004. After Method: Mess in Social Science Research. New York: Routledge.

Law, J. 2009. Actor Network Theory and Material Semiotics. In B. S. Turner (ed.) The New Blackwell Companion to Social Theory. Oxford: Blackwell.

Lely, J. and Saunders, J. 2012. Word Events. London: Continuum.

Lippard, L. and Chandler, J. 1967. The Dematerialization of Art. Art International 12(2): 31-6.

Lucier, A. 1995. Reflections: Interviews, Scores, Writings 1965-1994. Cologne: Musik Texte.
Murphy, J., Kapur, A. and Carnegie, D. 2012. Musical Robotics in a Loudspeaker World: Developments in Alternative Approaches to Localization and Spatialization. Leonardo Music Journal 22: 41-8.

Nyman, M. 1999. Experimental Music: Cage and Beyond, 2nd edn. Cambridge: Cambridge University Press.

Reich, S. 1969. Music as a Gradual Process. In C. Cox and D. Warner (eds.) Audio Culture: Readings in Modern Music. London and New York: Continuum.

Rose, E. 2013. Translating Transformations: Object Based Sound Installations. Leonardo Music Journal 23: 65-9.

Schoenherr, S. 2001. Loudspeaker History. www.aes.org/ aeshc/docs/recording.technology.history/loudspeaker.html (accessed July 2016).

Sterne, J. 2003. The Audible Past. Durham, NC: Duke University Press.

Toop, D. 1999. Humans, Are They Really Necessary? In R. Young (ed.) Undercurrents: The Hidden Wiring of Modern Music. London and New York: Continuum, 2002.

Tudor, D. 1988. Interview by Teddy Hultburg, Dussledorf, 17-18 May. The David Tudor Pages. http://davidtudor. org/Articles/hultberg.html (accessed October 2014).

Valle, A. 2013. Making Acoustic Computer Music: The Rumentarium Project. Organised Sound 18(3): 242-54.

Wilson, D. 2012. Miraculous Agitations: On the Uses of Chaotic, Non-Linear and Emergent Behavior in Acoustic Vibrating Physical Systems. Leonardo Music Journal 22: 35-40. 